\title{
Interaction between Alizarin and Human Serum Albumin by Fluorescence Spectroscopy
}

\author{
Feng Ge, ${ }^{\dagger}$ Lixiang Jiang, Diqiu LiU, and Chaoyin Chen \\ School of Life Science and Technology, Kunming University of Science and Technology, Kunming, \\ Yunnan 650224, P. R. China
}

\begin{abstract}
The binding properties on alizarin to human serum albumin (HSA) have been studied for the first time using fluorescence spectroscopy in combination with UV-visible absorbance spectroscopy. The results showed that alizarin strongly quenched the intrinsic fluorescence of HSA through a static quenching procedure, and non-radiation energy transfer occurred within the molecules. The number of binding sites was 1 , and the efficiency of Förster energy transfer provided a distance of $1.83 \mathrm{~nm}$ between tryptophan and alizarin binding site. $\Delta H^{\theta}, \Delta S^{\theta}$ and $\Delta G^{\theta}$ were obtained based on the quenching constants and thermodynamic theory $\left(\Delta H^{\theta}<0, \Delta S^{\theta}>0\right.$ and $\left.\Delta G^{\theta}<0\right)$. These results indicated that hydrophobic and electrostatic interactions are the main binding forces in the alizarin-HSA system. In addition, the results obtained from synchronous fluorescence spectra and three-dimensional fluorescence spectra showed that the binding of alizarin with HSA could induce conformational changes in HSA.
\end{abstract}

(Received June 30, 2010; Accepted September 25, 2010; Published January 10, 2011)

Alizarin (molecular structure, Fig. 1; formula, $\mathrm{C}_{14} \mathrm{H}_{8} \mathrm{O}_{4}$; molecular weight, 240.23), which is the principal coloring matter of the madder plant (Rubia tinctorum L.), ${ }^{1}$ known historically for its wide use as a natural dyestuff throughout the eastern and western continents. However, alizarin is a recalcitrant dye that is both mutagenic and carcinogenic. ${ }^{2}$ The high incidence of cancer among workers in the dye and chemical industries has been associated with exposure to anthraquinone dyes, ${ }^{3,4}$ and it is important to investigate the molecular mechanisms involved in these processes. ${ }^{5}$

Protein is an important chemical substance in our lives and the major target of many types of chemicals in the body. Knowledge of the interaction mechanisms between chemicals and plasma proteins is of crucial importance for us to understand the pharmacology or toxicology of a chemical. Chemical binding influences the distribution, excretion, metabolism, and interaction with the target tissues. Chemicals are mainly transported by human serum albumin (HSA). HSA is the most abundant plasma protein, which accounts for approximately $60 \%$ of the total proteins corresponding to a concentration of $40 \mathrm{mg} \mathrm{mL}^{-1}$ in the blood. ${ }^{6}$ The three-dimensional structure of HSA was determined through X-ray crystallographic measurements. ${ }^{7}$ HSA is a globular protein composed of<smiles>O=C1c2ccccc2C(=O)c2c1ccc(O)c2O</smiles>

Fig. 1 Molecular structure of alizarin.

$\dagger$ To whom correspondence should be addressed.

E-mail: gefeng79@yahoo.com.cn
585 amino acid residues in three homologous $\alpha$-helical domains (I, II, III). Each domain contains 10 helices, and is divided into six antiparallel helices and two subdomains (A and B). There is only one tryptophan located at position 214 along the chain, which is in subdomain II A of HSA. $^{8}$ Investigating the interaction of chemicals to HSA can elucidate the properties of chemical-protein complex, since it may provide useful information about the structural features that determine the therapeutic effectiveness of chemicals. Therefore, it has become an important research field in life sciences, chemistry, and clinical medicine.

There have been some work to study the interaction of chemicals with proteins by fluorescence spectroscopy, ${ }^{9}$ UV-spectrophotometry, ${ }^{10}$ circular dichroism spectroscopy (CD),${ }^{11}$ electrochemistry, ${ }^{12}$ equilibrium dialysis, ${ }^{13}$ attenuated total reflectance-Fourier transform infrared (ATR-FTIR), ${ }^{14}$ Raman spectroscopy, ${ }^{15}$ and nuclear magnetic resonance (NMR). ${ }^{16}$ Fluorescence quenching is a powerful method to study molecular interactions involving proteins, because it is sensitive, rapid and relatively easy to use. Fluorescence spectroscopy is essentially a probe technique sensing changes in the local environment of the fluorophore, which helps to understand albumin's binding mechanisms to compounds, and provide clues to concerning the nature of the binding phenomenon. ${ }^{17}$

To study the binding properties of hydrophobic molecules to proteins, a fluorometric titration using 8-anilino-1-naphthalenesulfonate (ANS) is popular because of its unique fluorescence characteristics. ANS fluoresces strongly in a hydrophobic environment; though it scarcely fluoresces in water, therefore, the change in the fluorescence intensity of ANS is a very sensitive indicator for the transfer of ANS from the bulk aqueous solution to the hydrophobic binding sites in protein. Competitive binding analysis using the fluorescence of ANS provides useful information on the binding properties of a target species. ${ }^{18}$

In this work, the binding of alizarin to HSA was studied by fluorescence and the UV-Vis spectrum. The binding mechanism 
between alizarin and HSA regarding the binding parameters, the thermodynamic functions, the binding distances and the effect of alizarin upon the conformation of HSA were investigated.

\section{Experimental}

\section{Reagents and chemicals}

Alizarin and 8-anilino-1-naphthalenesulfonic acid sodium salt (ANS-Na) were obtained from Sigma-Aldrich Co. (USA). HSA (fatty acid free $<0.05 \%$ ) was purchased from Sigma-Aldrich Co. (USA); it was used without further purification, and its molecular weight was assumed to be 66500 to calculate the molar concentrations. A Tris- $\mathrm{HCl}\left(0.05 \mathrm{~mol} \mathrm{~L}^{-1}\right)$ buffer solution containing $0.1 \mathrm{~mol} \mathrm{~L}^{-1} \mathrm{NaCl}$ was used to keep the $\mathrm{pH}$ of the solution at 7.40. A HSA stock solution of $1.0 \times 10^{-5} \mathrm{~mol} \mathrm{~L}^{-1}$ was prepared with the Tris- $\mathrm{HCl}$ buffer solution and kept in the dark at $4^{\circ} \mathrm{C}$. A stock solution $\left(5.0 \times 10^{-4} \mathrm{~mol} \mathrm{~L}^{-1}\right)$ of alizarin was prepared in methanol. All other chemicals were of analytical grade, and double distilled water was used throughout the experiments. All stock solutions were stored at $4^{\circ} \mathrm{C}$.

\section{Apparatus}

Fluorescence measurements were performed with an LS50B spectrofluorometer (Perkin Elmer, USA). The widths of both the excitation slit and the emission slit were set at $5 \mathrm{~nm}$. The UV-vis spectrum was recorded on a U-2000 spectrophotometer (Pharmacia, Sweden). An electronic thermoregulating water bath (NTT-2100, EYELA, Japan) was used to control the temperature of the samples.

\section{Procedures}

Fluorescence measurements were carried out by the successive addition of a $5.0 \times 10^{-4} \mathrm{~mol} \mathrm{~L}^{-1}$ stock solution of alizarin to a fixed amount of HSA (to give a final concentration of $\left.1.0 \times 10^{-6} \mathrm{~mol} \mathrm{~L}^{-1}\right)$ in each test tube. The final volume was made up to $5.0 \mathrm{~mL}$ with $\mathrm{pH} 7.40$ Tris- $\mathrm{HCl}$ buffer. Thus, a series of solutions containing different amounts of alizarin and a definite amount of HSA were obtained; they were mixed up and held in a thermostated water bath for 10 min before a fluorescence measurement. Fluorescence emission spectra were read in the range of wavelength from 300 to $450 \mathrm{~nm}$ using an excitation wavelength of $280 \mathrm{~nm}$. All experiments were performed at three temperatures $(298,310$ and $323 \mathrm{~K})$. The temperature of the sample was kept by recycling water throughout the experiment. Synchronous fluorescence spectra of HSA $\left(1.0 \times 10^{-6} \mathrm{~mol} \mathrm{~L}^{-1}\right)$ in the presence of alizarin $\left(2.0 \times 10^{-6}\right.$ to $\left.1.9 \times 10^{-5} \mathrm{~mol} \mathrm{~L}^{-1}\right)$ were recorded. The wavelength ranges of synchronous scanning were from 250 to $350 \mathrm{~nm}(\Delta \lambda=15,60 \mathrm{~nm})$ at $298 \mathrm{~K}$.

To estimate the binding site of alizarin with HSA, an ANS-HSA solution $2.97 \mathrm{~cm}^{3}$ was titrated with an appropriate concentration of a $0.03-\mathrm{cm}^{3}$ alizarin methanol solution. It was confirmed that this methanol concentration did not affect the fluorescence intensity of ANS-HSA. The fluorescence intensity of ANS was measured with an excitation wavelength of $373 \mathrm{~nm}$. The emission spectra of ANS were recorded between 465 and $485 \mathrm{~nm}$ at $298 \mathrm{~K}$.

\section{Results and Discussion}

Interaction between alizarin and HSA

Fluorescence quenching is a decrease of the quantum yield of fluorescence from a fluorophore induced by a variety of

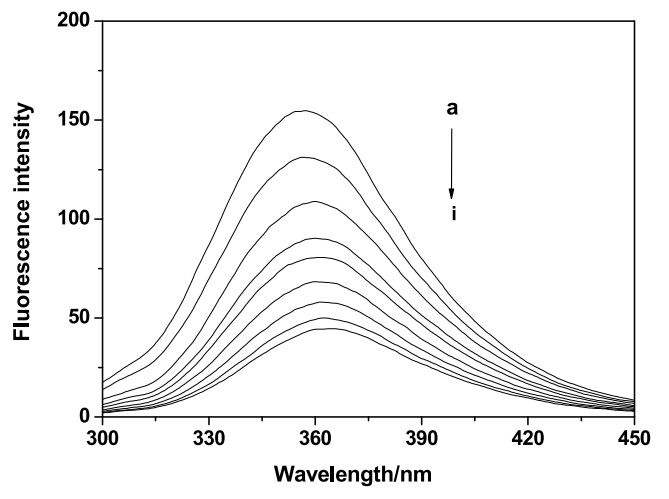

Fig. 2 Fluorescence emission spectra of HSA-alizarin system excited at $280 \mathrm{~nm}(\mathrm{pH} 7.40, T=298 \mathrm{~K})$. (a) $1 \times 10^{-6} \mathrm{~mol} \mathrm{~L}^{-1} \mathrm{HSA}$; (b) - (i) $1 \times 10^{-6} \mathrm{~mol} \mathrm{~L}^{-1} \mathrm{HSA}$ in the presence of $0.20,0.40,0.60,0.80$, $1.0,1.3,1.6$ and $1.9 \times 10^{-5} \mathrm{~mol} \mathrm{~L}^{-1}$ alizarin, respectively.

molecular interactions with quencher molecules, including exciting-state reactions, molecular rearrangements, energy transfer, ground-state complex formation and collisional quenching processes. Quenching can be classified as either dynamic or static quenching by different mechanisms. Dynamic quenching results from collisions between the fluorophore and the quencher, and static quenching is due to the formation of a ground-state complex between the fluorophore and the quencher. In general, dynamic and static quenching can be distinguished by their different dependence on the temperature and the viscosity. The quenching rate constants decrease with increasing temperature for static quenching, but the reverse effect is observed for dynamic quenching. ${ }^{19}$ The fluorescence spectrum of HSA in the presence of different amounts of alizarin was recorded in the range of $300-450 \mathrm{~nm}$ upon excitation at $280 \mathrm{~nm}$ (Fig. 2). As can be seen from Fig. 2, the fluorescence intensity of HSA gradually decreased upon increasing the concentration of alizarin, indicating the binding of alizarin to HSA. The maximum wavelength of HSA shifted from 355 to $365 \mathrm{~nm}$ after the addition of alizarin. A red shift of the fluorescence spectrum indicated that tryptophane residue was brought to a more hydrophilic environment in the alizarin-HSA system, and the structure of hydrophobic subdomain where tryptophane placed was not compact, and the segment of polypeptide changed its conformation to a more extended state after the addition of alizarin. ${ }^{9}$

In order to confirm the quenching mechanism, the fluorescence quenching data were analyzed by the Stern-Volmer, ${ }^{20}$

$$
\frac{F_{0}}{F}=1+K_{\mathrm{q}} \tau_{0}[Q]=1+K_{\mathrm{sv}}[Q]
$$

where $F_{0}$ and $F$ are the steady-state fluorescence intensities in the absence and presence of a quencher, respectively. $K_{\mathrm{q}}$ is the quenching rate constant of the bimolecules, $\tau_{0}$ the average lifetime of the biomolecule without a quencher $\left(\tau_{0}=10^{-8} \mathrm{~s}\right),{ }^{19}$ $K_{\text {sv }}$ the Stern-Volmer quenching constant, and $[Q]$ the concentration of the quencher. Figure 3 displays Stern-Volmer plots of the quenching of the HSA fluorescence by alizarin at different temperatures. The plot shows that within the investigated concentrations, the results agree with the Stern-Volmer Eq. (1). Table 1 summarizes the calculated $K_{\mathrm{sv}}$ and $K_{\mathrm{q}}$ values at each temperature studied. The results showed that the values of the Stern-Volmer quenching constants, $K_{\mathrm{sv}}$ and 


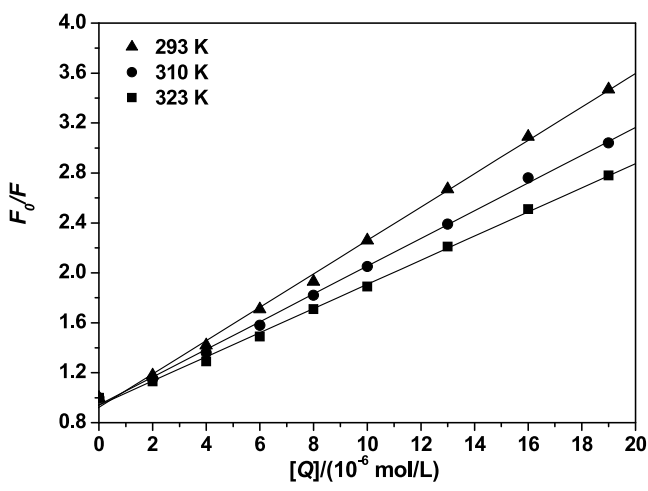

Fig. 3 Stern-Volmer plot of HSA-alizarin system at different temperatures $(298 \mathrm{~K}, \quad r=0.9989 ; 310 \mathrm{~K}, \quad r=0.9991 ; 323 \mathrm{~K}$, $r=0.9989) . C_{\mathrm{HSA}}=1 \times 10^{-6} \mathrm{~mol} \mathrm{~L}^{-1} ; \lambda_{\mathrm{ex}}=280 \mathrm{~nm} ; \mathrm{pH} 7.40$.

Table 1 Quenching and dissociation constants of HSA with alizarin at different temperatures

\begin{tabular}{ccccc}
\hline$T / \mathrm{K}$ & $K_{\text {sv }} / 10^{5} \mathrm{~L} \mathrm{~mol}^{-1}$ & $K_{\mathrm{q}} / 10^{12} \mathrm{~L} \mathrm{~mol}^{-1} \mathrm{~s}^{-1}$ & $R^{\mathrm{a}}$ & $\mathrm{SD}^{\mathrm{b}}$ \\
\hline 298 & 1.34 & 1.34 & 0.9989 & 0.042 \\
310 & 1.11 & 1.11 & 0.9991 & 0.032 \\
323 & 0.967 & 0.967 & 0.9989 & 0.031 \\
\hline
\end{tabular}

a. $R$ is the correlation coefficient

b. SD is the standard deviation for the $K_{\mathrm{sv}}$ values.

$K_{\mathrm{q}}$, decreased with increasing temperatures, which indicated that the possible quenching mechanism of the fluorescence of HSA by alizarin was a static quenching type. On the other hand, the values of $K_{\mathrm{q}}$ were much greater than the maximum scatter collision quenching constant $\left(2.0 \times 10^{10} \mathrm{~mol} \mathrm{~L}^{-1} \mathrm{~S}^{-1}\right)$, which revealed that the probable quenching mechanism of the alizarin-HSA interaction was initiated by complex formation rather than by dynamic collisions. ${ }^{8}$ This demonstrates that the fluorescence quenching is not the result of dynamic collision quenching, but rather a consequence of static quenching.

\section{Calculation of binding constant}

When small molecules bind independently to a set of equivalent sites on a macromolecule, the binding constant $\left(K_{\mathrm{A}}\right)$ and the numbers of binding sites $(n)$ can be determined by the following: ${ }^{21,22}$

$$
\log \frac{F_{0}-F}{F}=\log K_{\mathrm{A}}+n \log [Q]
$$

where $F_{0}$ and $F$ are the fluorescence intensities before and after the addition of the quencher; $[Q]$ is the quenching concentration. Figure 4 shows plots of $\log \left(F_{0} / F-1\right)$ versus $\log [Q]$ for the alizarin-HSA system at different temperatures; the calculated results are presented in Table 2. As can be seen from Table 2, the binding constants, $K_{\mathrm{A}}$, decreased with increasing the temperature, which may indicate forming an unstable compound, and the unstable compound would be partly decomposed with the rising temperature. The values of $n$ at the experimental temperatures were approximately equal to 1 , which indicated that there was one class of binding sites to alizarin in HSA.

\section{$U V$-Vis absorption spectroscopy}

The UV-Vis absorption measurenment is a simple, but

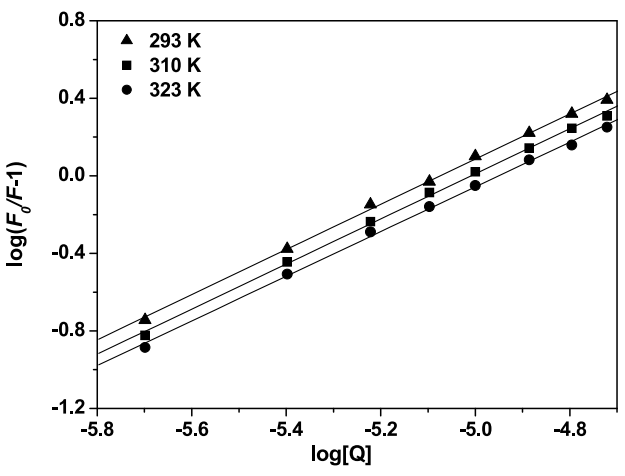

Fig. 4 The plot of $\log \left(F_{0} / F-1\right)$ vs. $\log [Q]$ at different temperatures $(298 \mathrm{~K}, r=0.9993 ; 310 \mathrm{~K}, r=0.9992 ; 323 \mathrm{~K}, r=0.9991) . \quad C_{\mathrm{HSA}}=$ $1 \times 10^{-6} \mathrm{~mol} \mathrm{~L}^{-1} ; \lambda_{\text {ex }}=280 \mathrm{~nm} ; \mathrm{pH} 7.40$.

Table 2 Binding parameters of alizarin-HSA at different temperatures

\begin{tabular}{ccccc}
\hline$T / \mathrm{K}$ & $K_{\text {sv }} / 10^{5} \mathrm{~L} \mathrm{~mol}^{-1}$ & $n$ & $R^{\mathrm{a}}$ & $\mathrm{SD}^{\mathrm{b}}$ \\
\hline 298 & 8.13 & 1.17 & 0.9989 & 0.042 \\
310 & 6.61 & 1.16 & 0.9991 & 0.032 \\
323 & 5.13 & 1.15 & 0.9989 & 0.031 \\
\hline
\end{tabular}

a. $R$ is the correlation coefficient.

b. SD is the standard deviation for the $K_{\mathrm{sv}}$ values.

effective, method in detecting complex formation. A complex formed between alizarin and HSA was evident from the data of the UV-Vis absorption spectra (Fig. 5). It can be seen that the intensity of the UV absorption of HSA changes with an alteration of the alizarin concentration. Moreover, red shift of the maximum peak of HSA at $210 \mathrm{~nm}$ was also noticed, probably due to complex formation between alizarin and HSA.

\section{Thermodynamic analysis and the nature of the binding force}

The binding forces between small molecular and biomacromolecules may include the hydrophobic force, electrostatic interactions, van der Waals interactions, hydrogen bonds, etc. ${ }^{14}$ The thermodynamic parameters of the binding reaction are the main evidence for confirming the binding force. For this purpose, the temperature-dependence of the binding constant was measured at three different temperatures $(298,310$ and $323 \mathrm{~K}$ ). If the enthalpy change $\left(\Delta H^{\theta}\right)$ does not vary significantly over the temperature range studied, its value and that of entropy change $\left(\Delta S^{\theta}\right)$ can be determined from the van't Hoff:

$$
\ln K=-\frac{\Delta H^{\theta}}{R T}+\frac{\Delta S^{\theta}}{R} .
$$

The free energy change $\left(\Delta G^{\theta}\right)$ of the binding reaction at different temperature was calculated from

$$
\Delta G^{\theta}=\Delta H^{\theta}-T \Delta S^{\theta},
$$

where $K$ is the binding constant at the corresponding temperature $T, R$ the gas constant, and $T$ the absolute temperature. There was a good linear relationship between $\ln K$ and $1 / T$ (Fig. 6). Table 3 gives the values of $\Delta H^{\theta}, \Delta S^{\theta}$ and $\Delta G^{\theta}$. The negative sign for $\Delta G^{\theta}$ means the spontaneity of the binding of alizarin 


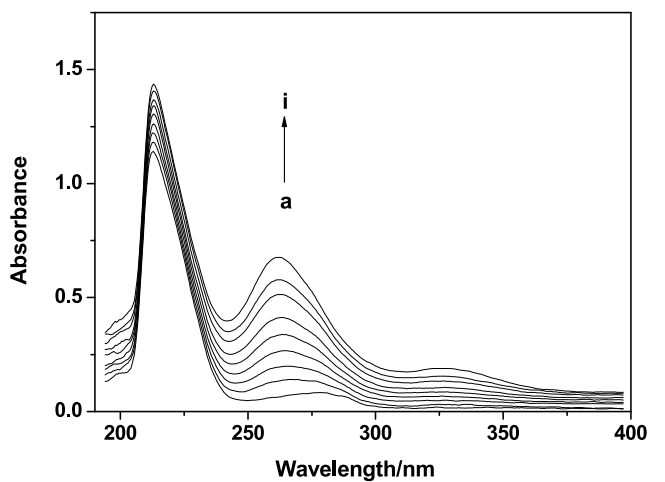

Fig. 5 Absorbance spectra of HSA in the presence of alizarin. (a) $1 \times 10^{-6} \mathrm{~mol} \mathrm{~L}^{-1} \mathrm{HSA}$; (b) - (i) $1 \times 10^{-6} \mathrm{~mol} \mathrm{~L}^{-1} \mathrm{HSA}$ in the presence of $0.20,0.40,0.60,0.80,1.0,1.3,1.6$ and $1.9 \times 10^{-5} \mathrm{~mol} \mathrm{~L}^{-1}$ alizarin, respectively. $\lambda_{\mathrm{ex}}=280 \mathrm{~nm} ; \mathrm{pH} 7.40 ; T=298 \mathrm{~K}$.

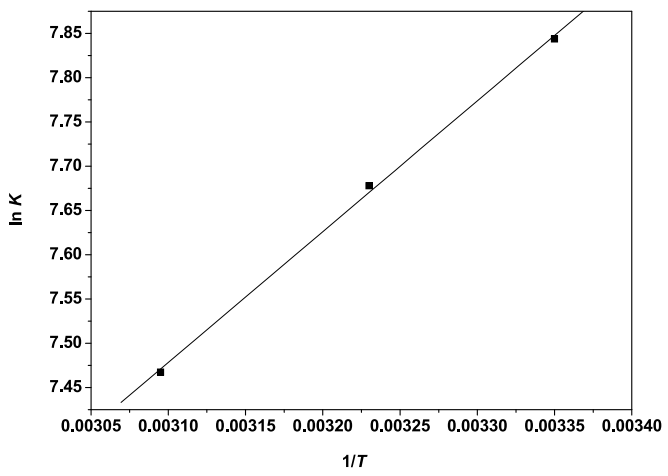

Fig. 6 Van't Hoff plot of HSA-alizarin $(\ln K=7.539+1814 / T$, $r=0.9990)$.

with HSA. Generally, a positive $\Delta S^{\theta}$ value is taken as typical evidence for a hydrophobic interaction, because the water molecules that are arranged in an orderly fashion around the ligand and protein acquire a more random configuration as a result of the hydrophobic reaction. In addition, a specific electrostatic interaction between ionic species in an aqueous solution is characterized by a positive $\Delta S^{\theta}$ value and a negative $\Delta H^{\theta}$ value. ${ }^{23}$ From the positive $\Delta S^{\theta}$ value and the negative $\Delta H^{\theta}$ value observed in the present study, it can be concluded that hydrophobic and electrostatic interactions are the main binding forces in the alizarin-HSA system.

\section{Distance measurement between alizarin and binding site}

According to the Förster non-radiative resonance energy transfer theory, ${ }^{24}$ the rate of energy transfer depends on (i) the relative orientation of the donor and the acceptor dipoles, (ii) the extent of overlap of the fluorescence emission spectrum of the donor with the absorption spectrum of the acceptor and (iii) the distance between the donor and the acceptor. Here, the donor and the acceptor were HSA and alizarin, respectively. The absorption spectrum of alizarin was recorded in the range of $350-500 \mathrm{~nm}$ in the $\mathrm{pH} 7.40$ Tris- $\mathrm{HCl}$ buffer. The emission spectrum of HSA was also recorded under the same condition. There was a spectral overlap between the fluorescence emission spectrum of HSA and the UV-Vis absorption spectrum of alizarin (Fig. 7). The efficiency of energy transfer $(E)$ is related
Table 3 Thermodynamic parameters of the alizarin-HSA binding process

\begin{tabular}{cccc}
\hline$T / \mathrm{K}$ & $\Delta H^{\theta} / \mathrm{kJ} \mathrm{mol}^{-1}$ & $\Delta S^{\theta} / \mathrm{J}(\mathrm{K} \mathrm{mol})^{-1}$ & $\Delta G^{\theta} / \mathrm{kJ} \mathrm{mol}^{-1}$ \\
\hline 298 & & & -33.76 \\
310 & -15.08 & 62.68 & -34.51 \\
323 & & & -35.33 \\
\hline
\end{tabular}

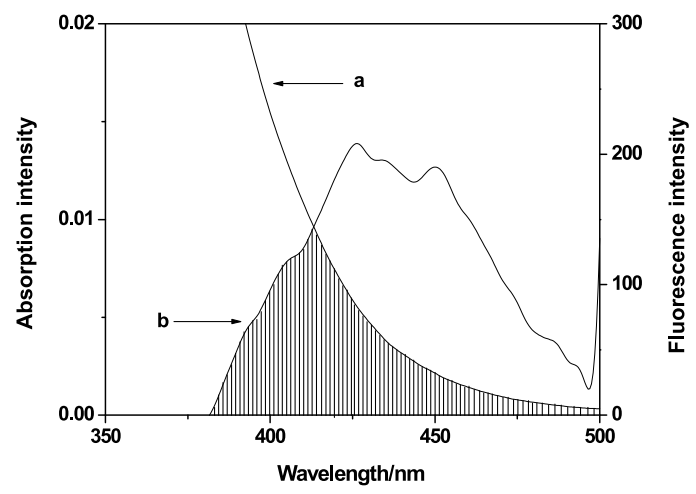

Fig. 7 Spectral overlap between the fluorescence emission spectrum of HSA (a) and the UV-Vis absorption spectrum of alizarin (b). $C_{\mathrm{HSA}}=$ $5.0 \times 10^{-6} \mathrm{~mol} \mathrm{~L}^{-1} ; C_{\text {alizarin }}=5.0 \times 10^{-6} \mathrm{~mol} \mathrm{~L}^{-1} ; \mathrm{pH} 7.40 ; T=298 \mathrm{~K}$.

to the distance $(R)$ between the donor and acceptor. $E$ could be calculated using

$$
E=1-\frac{F}{F_{0}}=\frac{R_{0}^{6}}{R_{0}^{6}+r^{6}},
$$

where $F_{0}$ and $F$ are the fluorescence intensities without and with alizarin, respectively; $r$ the distance between the acceptor and the donor, and $R_{0}$ the critical distance when the transfer efficiency is $50 \%$,

$$
R_{0}^{6}=8.8 \times 10^{-25} k^{2} n^{-4} \varphi J
$$

where $k^{2}$ is the spatial orientation factor between the emission dipole of the donor and the absorption dipole of the acceptor, $n$ the average refractive index of the medium, $\varphi$ the fluorescence quantum yield of the donor and $J$ the overlap integral of the fluorescence emission spectrum of the donor and the absorption spectrum of the receptor. $J$ is given by

$$
J=\frac{\sum F(\lambda) \varepsilon(\lambda) \lambda^{4} \Delta \lambda}{\sum F(\lambda) \Delta \lambda},
$$

where $F(\lambda)$ is the fluorescence intensity of the donor in the wavelength range $\lambda$ to $\lambda+\Delta \lambda ; \varepsilon(\lambda)$ the molar absorption coefficient of the acceptor at $\lambda$. In the present case, $k^{2}=2 / 3$, $n=1.336, \varphi=0.15 .^{2}$ According to Eqs. (5) - (7), the values of the parameters were found to be $J=4.829 \times 10^{-16} \mathrm{~cm}^{3} \mathrm{~L} \mathrm{~mol}^{-1}$, $R_{0}=1.54 \mathrm{~nm}, E=0.26$ and $r=1.83 \mathrm{~nm}$. The distance between donor and acceptor is less than $8 \mathrm{~nm},{ }^{25}$ which implied that the energy transfer from HSA to alizarin occurred with high probability. This accorded with the conditions of the Förster non-radiative resonance energy transfer theory, and indicated again a static quenching between HSA and alizarin. 


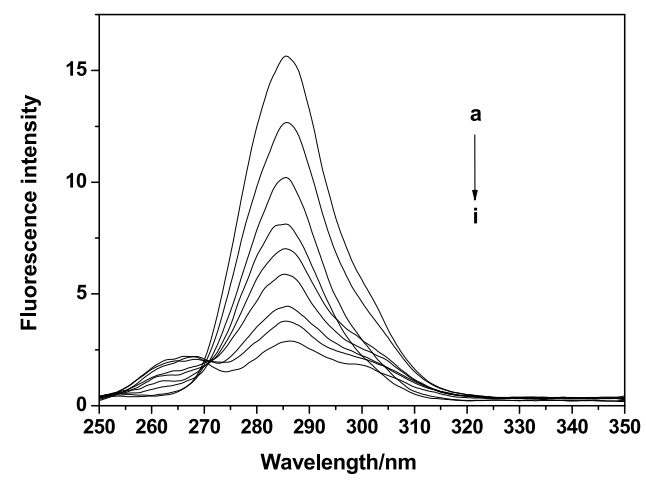

Fig. 8 Synchronous fluorescence spectrum $(\Delta \lambda=15 \mathrm{~nm}, \mathrm{pH} 7.40$, $T=298 \mathrm{~K}$ ). (a) - (i): same as in Fig. 5.

\section{Investigation on the conformational change of the complex}

Synchronous fluorescence spectroscopy was used to characterize complex mixtures, since it can provide fingerprints of complex samples. ${ }^{26,27}$ It gives information about the molecular environment in the vicinity of the chromosphere molecules, and has several advantages, such as sensitivity, spectral simplification and avoiding different perturbing effects. When $\Delta \lambda$ between the excitation wavelength and the emission wavelength were set at 15 or $60 \mathrm{~nm}$, the synchronous fluorescence could provide characteristic information of the tyrosine residues or tryptophan residues in HSA, respectively. ${ }^{2,8}$ By investigating the synchronous fluorescence spectra of tyrosine residues and tryptophan residues, we could explore the conformational changes of HSA.

The effect of alizarin on the synchronous fluorescence spectrum of HSA is shown in Figs. 8 and 9. It can be seen from Fig. 8 that the maximum emission wavelength of tyrosine residues does not have a significant shift. In contrast, an obvious red shift (from 283 to $289 \mathrm{~nm}$ ) of tryptophan residues (Fig. 9) was observed, which indicated that the polarity around the tryptophan residues was increased and the hydrophobicity was decreased, yet the microenvironment around the tyrosine residues had no discernable change during the binding process. ${ }^{2}$ Moreover, the fluorescence intensity decreased regularly with the addition of alizarin, which further demonstrated the occurrence of fluorescence quenching in the binding process. It has also been shown in Fig. 10 that the slope was higher when $\Delta \lambda$ was $60 \mathrm{~nm}$, indicating that a significant contribution of the tryptophan residue in the fluorescence of HSA, alizarin was closer to the tryptophan residue compared to the tyrosine residue.

ANS has been frequently used to study the binding properties of hydrophobic species to albumins. ${ }^{28,29}$ Some papers indicated that there were three fluorescence-active ANS binding sites in HSA. $^{30,31}$ The ANS binds most preferentially to the Trp-HSA site compared to the other two sites in HSA, and Trp-HSA is located in the subdomain II A. ${ }^{31}$ In a competitive binding assay of alizarin with ANS-HSA, it decreased about $40 \%$ of the initial intensity of the ANS-HSA fluorescence. This value corresponds to the release of one point two ANS among the total three binding sites in HSA. It can be concluded from these results that, under the present condition, alizarin binds mainly to the Trp-HSA site.

The three-dimensional fluorescence spectrum has become a popular technique in recent years. The outstanding advantage of three-dimensional fluorescence spectra is that information regarding the fluorescence characteristics can be entirely

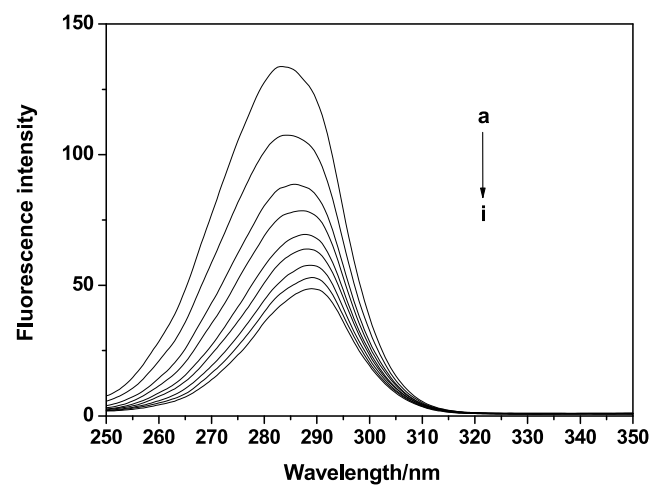

Fig. 9 Synchronous fluorescence spectrum $(\Delta \lambda=60 \mathrm{~nm}, \mathrm{pH} 7.40$, $T=298$ K). (a) - (i): same as in Fig. 5.

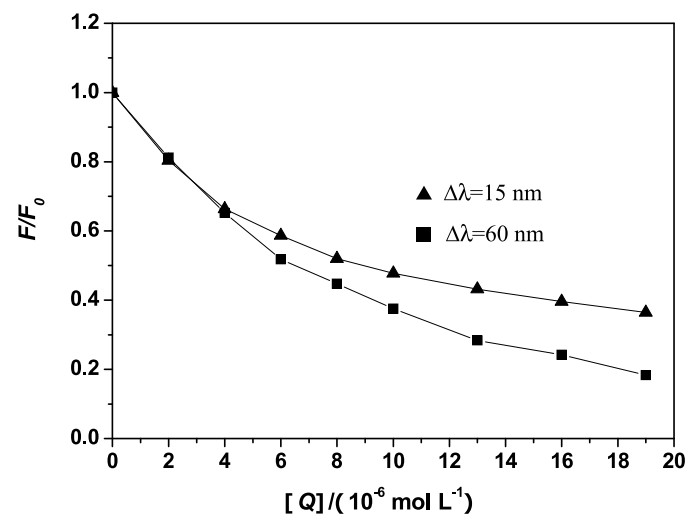

Fig. 10 Quenching of the HSA synchronous fluorescence by alizarin. $C_{\mathrm{HSA}}=1.0 \times 10^{-6} \mathrm{~mol} \mathrm{~L}^{-1} ; \mathrm{pH} 7.40 ; T=298 \mathrm{~K}$.

acquired by changing the excitation and emission wavelengths simultaneously. ${ }^{32}$ The three-dimensional fluorescence spectra of HSA and alizarin-HSA complex are shown in Fig. 11. As shown in Fig. 11, peak 1 is the Rayleigh scattering peak $\left(\lambda_{\mathrm{ex}}=\right.$ $\left.\lambda_{\mathrm{em}}\right){ }^{33}$ The fluorescence intensity of peak 1 increased with the addition of alizarin. A possible reason is that an alizarin-HSA complex came into being after the addition of dye, increasing the diameter of the macromolecule, which in turn resulted in an enhanced scattering effect. Peak 2 mainly reveals the spectral behavior of the Trp and Tyr residues. The reason is that when HSA is excited at $280 \mathrm{~nm}$, it mainly reveals the intrinsic fluorescence of the Trp and Tyr residues. Beside peak 2, there is another fluorescence peak $3\left(\lambda_{\mathrm{ex}}=230.0 \mathrm{~nm}, \lambda_{\mathrm{em}}=340.0 \mathrm{~nm}\right)$ that mainly reflects the fluorescence spectral behavior of the polypeptide backbone structure of HSA. ${ }^{34}$ The fluorescence intensity of peak 3 decreased after the addition of alizarin, which means that the peptide strands structure of HSA has been changed. Analyzing from the fluorescence intensity changes of peak 2 and peak 3, which decrease, but to a different degree: in the absence and presence of alizarin, the intensity ratio of peak 2 and peak 3 was 1.30:1 and 1.40:1, respectively. The decrease in the fluorescence intensity of the two peaks in combination with synchronous fluorescence and UV-Vis absorption results indicated that the interaction of dye with HSA induced a secondary structure change of HSA. The above phenomenon and analyzing peak 2 and peak 3 revealed that the binding of alizarin to HSA induced some microenvironment and conformational changes in HSA. 

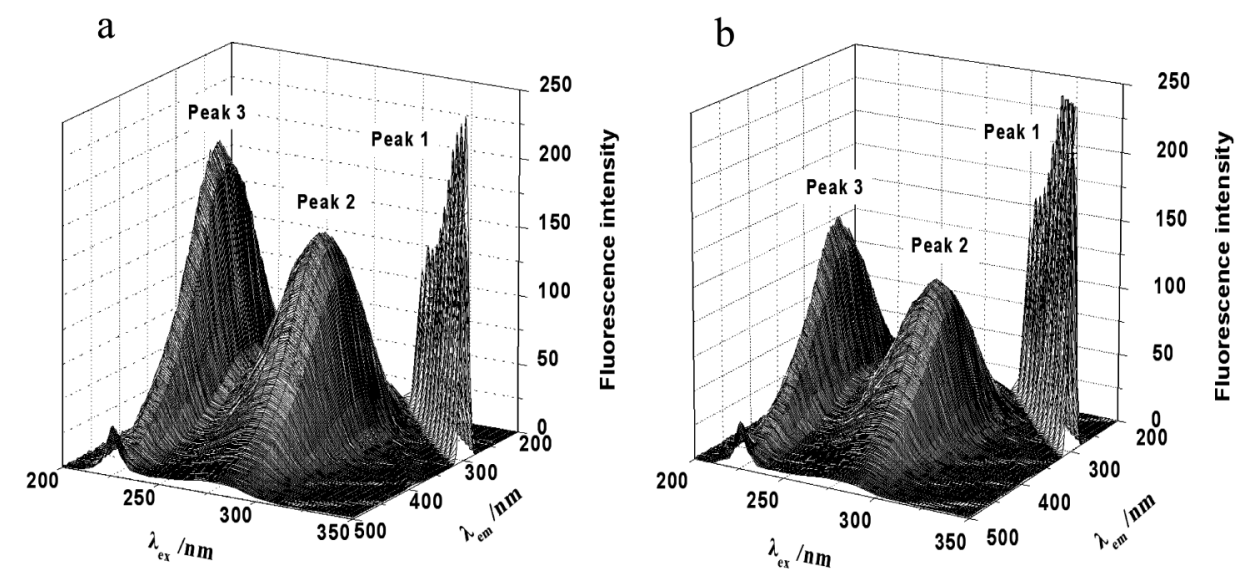

Fig. 11 Three-dimensional fluorescence spectra of HSA (a) and the alizarin-HSA system (b). (a) $C_{\mathrm{HSA}}=$ $1.2 \times 10^{-6} \mathrm{~mol} \mathrm{~L}^{-1}, C_{\text {alizarin }}=0$; (b) $C_{\mathrm{HSA}}=1.0 \times 10^{-6} \mathrm{~mol} \mathrm{~L}^{-1}, C_{\text {alizarin }}=5.0 \times 10^{-6} \mathrm{~mol} \mathrm{~L}^{-1} ; \mathrm{pH} 7.40 ; T=298 \mathrm{~K}$.

The results of this paper may be important, which may provide information about the structural features that determine the pharmacology or toxicology of chemicals. A better understanding of pharmacokinetics such as drug metabolism, excretion and distribution can be obtained by the binding parameters in a drug-protein system. In addition, the study on binding sites will help to synthesize drugs targeted to perform specific tasks in the human body. Furthermore, in order to obtain more informations about the interactions between alizarin and biomacromolecules, the interactions of alizarin with DNA will be studied in our next work.

\section{Acknowledgements}

This work is supported by the National Natural Science Foundation of China (No. 31060044), the Foundation of Yunnan Province (No. 2009ZC046M) and the National Foundation of Innovative Experiment for College Student of China (No. 091067444).

\section{References}

1. C. Ahn and S. K. Obendorf, Text. Res. J., 2004, 74, 949.

2. F. Ding, J. L. Huang, J. Lin, Z. Y. Li, F. Liu, Z. Q. Jiang, and Y. Sun, Dyes Pigm., 2009, 82, 65.

3. T. C. Marrs, H. F. Colgrave, P. Rice, J. A. G. Edginton, and B. Morris, J. Hazard. Mater., 1989, 21, 73.

4. R. H. Jaskot and D. L. Costa, J. Appl. Toxicol., 1994, 22, 103.

5. Č. Novotný, N. Dias, A. Kapanen, K. Malachová, M. Vándrovcová, and M. Itävaara, Chemosphere, 2006, 63, 1436.

6. T. Peters, "All about Albumin: Biochemistry, Genetics and Medical Applications", 1996, Academic Press, San Diego.

7. M. H. Xiao and D. C. Carter, Nature, 1992, 358, 209.

8. G. W. Zhang, Q. M. Que, J. H. Pan, and J. B. Guo, J. Mol. Struct., 2008, 881, 132.

9. S. Y. Bi, Y. T. Sun, C. Y. Qiao, H. Q. Zhang, and C. M. Liu, J. Lumin., 2009, 129, 541.

10. S. Baroni, M. Mattu, A. Vannini, R. Cipollone, S. Aime, P. Ascenzi, and M. Fasano, Eur. J. Biochem., 2001, 268, 6214.

11. W. Y. He, Y. Li, J. N. Tian, H. X. Liu, Z. D. Hu, and X. G.
Chen, J. Photochem. Photobiol., A, 2005, 174, 53.

12. Y. H. Wu, X. B. Ji, and S. S. Hu, Bioelectrochemistry, 2004, 64, 91.

13. I. Girard and S. Ferry, J. Pharm. Biomed. Anal., 1996, 14, 583.

14. J. H. Tang, F. Luan, and X. G. Chen, Bioorg. Med. Chem., 2006, 14, 3210.

15. X. C. Shen, H. Liang, J. H. Guo, C. Song, X. W. He, and Y. Z. Yuan, J. Inorg. Biochem., 2003, 95, 124.

16. A. Sułkowski, J. Równicka, J. Pożycka, B. Bojko, and W. W. Sułkowski, J. Mol. Struct., 2005, 744, 775.

17. D. Silva, C. M. Cortez, C. B. Jayme, and S. Loura, Toxicol. Lett., 2004, 147, 53.

18. E. C. Santos and A. A. Spector, Biochemistry, 1972, 11, 2299.

19. J. R. Lakowicz, "Principles of Fluorescence Spectroscopy", 2nd ed., 1999, Plenum Press, New York.

20. B. Zhou, Z. D. Qi, Q. Xiao, J. X. Dong, Y. Z. Zhang, and Y. Liu, J. Biochem. Biophys. Methods, 2007, 70, 743.

21. B. Ahmad, S. Parveen, and R. H. Khan, Biomacromolecules, 2006, 7, 1350.

22. Y. He, Y. W. Wang, L. F. Tang, H. Liu, W. Chen, Z. L. Zheng, and G. L. Zou, J. Fluoresc., 2008, 18, 433.

23. P. D. Ross and S. Subramanian, Biochemistry, 1981, 20, 3096.

24. T. Förster and O. Sinanoglu, in "Modern Quantum Chemistry", 1966, Vol. III, Acacemic Press, New York, 93.

25. S. Weiss, Science, 1999, 238, 1676.

26. J. B. F. Lloyd, J. Forensic Sci. Soc., 1971, 11, 235.

27. F. Ge, C. Y. Chen, D. Q. Liu, B. Y. Hang, X. F. Xiong, and S. L. Zhao, J. Lumin., 2010, 130, 168.

28. L. Stryer, J. Mol. Biol., 1965, 13, 482.

29. K. Takehara, Y. Morinaga, S. Nakashima, S. Matsuoka, H. Kamaya, and I. Ueda, Anal. Sci., 2006, 22, 1571.

30. M. Cardamone and N. K. Puri, Biochem. J., 1992, 282, 589.

31. K. Takehara, K. Yuki, M. Shirasawa, S. Yamasaki, and S. Yamada, Anal. Sci., 2009, 25, 115.

32. M. J. Rodríguez-Cuesta, R. Boqué, F. X. Rius, D. P. Zamora, M. M. Galera, and A. G. Frenich, Anal. Chim. Acta, 2003, 491, 47.

33. Y. Z. Zhang, X. X. Chen, J. Dai, X. P. Zhang, Y. X. Liu, and Y. Liu, Luminescence, 2008, 23, 150.

34. Y. Q. Wang, B. P. Tang, H. M. Zhang, Q. H. Zhou, and G. C. Zhang, J. Photochem. Photobiol., B, 2009, 94, 183. 